\title{
Association of environmental chemicals \& estrogen metabolites in children
}

\author{
Erin Speiser Ihde ${ }^{1 *}$, Ji Meng Loh² and Lawrence Rosen ${ }^{1}$
}

\begin{abstract}
Background: The prevalence of pediatric hormonal disorders and hormonally-sensitive cancers are rising. Chemicals including bisphenol A (BPA), phthalates, parabens, 4-nonylphenol (4NP) and triclosan have been linked to disruption of endocrine pathways and altered hormonal status in both animal and human studies. Additionally, changes in estrogen metabolism have been associated with pediatric endocrine disorders and linked to estrogendependent cancers. The main objective of the study was to measure the presence of these environmental chemicals in prepubescent children and assess the relationship between chemical metabolites and estrogen metabolism.
\end{abstract}

Methods: 50 subjects ( 25 male, 25 female) were recruited from the principal investigator's existing patient population at his pediatric primary care office. The first 5 boys and 5 girls in each age group (4 through 8 years old inclusive) who presented for annual examinations were included, as long as they were Tanner Stage I (prepubertal) on physical exam, without diagnosis of hormonally-related condition and/or cancer and able to give a urine sample. Urine samples were collected in glass containers for analysis of chemical and estrogen metabolites. Study kits and lab analysis were provided by Genova Diagnostics (Duluth, GA).

Summary statistics for the concentrations of each chemical metabolite as well as estrogen metabolites were computed (minimum, maximum, median and inter-quartile range) for males only, for females only and for all subjects. Comparisons between groups (e.g. males v. females) were assessed using the nonparametric Wilcoxon test, since the data was skewed. The correlation between concentrations of chemical metabolites and estrogen metabolites in prepubescent children were examined by the Spearman's correlation coefficient ( $\rho)$.

Results: $100 \%$ of subjects had detectible levels of at least five chemicals in their urine, and $74 \%$ had detectible levels of eight or more chemicals. Of note, $100 \%$ of subjects demonstrated presence of at least one chemical in each class of BPA, phthalates, and parabens. $28 \%$ of subjects had measurable levels of 4NP. No associations were found between the urine levels of chemicals and estrogen metabolites.

Conclusions: Endocrine disrupting environmental chemicals were detected in all children in the study, with measurable levels of 4NP in nearly $1 / 3$ of subjects. This is the first known published study of 4NP levels in American children. No associations were found between the urine levels of chemicals tested and estrogen metabolites. The presence of multiple chemicals in a majority of children's urine coupled with increasing prevalence of pediatric hormonal disorders warrants further research to elucidate potential causal mechanisms in pre- and post-pubertal children.

Keywords: BPA, Bisphenol A, Phthalates, 4-nonylphenol, Parabens, Triclosan, Pediatric, Puberty, Endocrine disruption, Estrogen

\footnotetext{
* Correspondence: eihde@HackensackUMC.org

${ }^{1}$ The Deirdre Imus Environmental Health Center ${ }^{\circledR}$, Hackensack University

Medical Center, 30 Prospect Ave, Research Building, Hackensack, NJ 07601,

USA

Full list of author information is available at the end of the article
} 


\section{Background}

In the United States, cancer is the leading cause of disease-related death among children and adolescents [1]. The incidence of pediatric cancers has increased at an annual rate of $0.6 \%$ since 1975 [2]. The largest contributor to this rise has been the germ cell, trophoblastic, and other gonadal tumor category, specifically testicular and ovarian germ cell tumors [2]. Additionally, studies have concluded that the incidence of altered pubertal onset is increasing, especially in girls [3]. Both developments have been linked to environmental factors, including exposure to endocrine disrupting chemicals [4-6]. Childhood exposure to these chemicals is widespread [4]. Of greatest concern are chemicals with potential for both endocrine disruption and carcinogenesis, including bisphenol A (BPA), phthalates, parabens, 4-nonylphenol (4NP) and triclosan [5-7].

BPA is commonly found in polycarbonate plastic products including baby bottles, water bottles, food containers, in the linings of metal food cans and in dental sealants and composites [8]. BPA has been found in over $90 \%$ of the U.S. population age six and over, with highest concentrations in children ages 6-11 [8]. Triclosan is commonly found in antibacterial hand soaps, toothpastes and household cleaning supplies [9]. Phthalate chemicals "soften" plastics to make them pliable. They are also found in personal care products, food, plastic toys and household dust [10]. Parabens are found in common personal care products including cosmetics and antiperspirants [10]. 4NP is one of the most common alkylphenols (APs) in industry, used in the production of cleaning products, plastics, rubber and personal care products, including hair products and spermicides [11]. APs are commonly used to make alkylphenolethoxylates (APEs), which are widely used surfactants and detergents [12, 13]. Despite widespread use, exposure data on 4NP is scarce. To our knowledge, there is no published data on 4NP exposures in American children [12, 14-20]. All of these five chemicals have been detected in a majority of the U.S. population [10, 12, 21].

BPA, phthalates, triclosan, parabens and 4NP have been linked to disruption of endocrine pathways and altered hormonal status in both animal and human studies. Two pediatric studies have linked phthalate exposure to precocious puberty in girls $[6,13]$ while another study found an association between urinary concentrations of high-molecular weight phthalate metabolites and later pubarche [22]. BPA, triclosan, 4NP and parabens have also been associated with endocrine disruption or hormonally derived cancers in vitro and in animal models [7, 23-27]. Changes in estrogen metabolism, specifically in the ratio of 16a-Hydroxyestrone (16a-OHE1) to 2-Hydroxyestrone (2-OHE1) metabolites, have been associated with hormone disruption and linked to estrogen-dependent cancers [28, 29].
Given the widespread exposure of humans to these chemicals and the concern about environmental factors causing endocrine disruption and cancer in children, our study team investigated whether the levels of urinary chemical metabolites in children are correlated with levels and ratios of urinary estrogen metabolites. Such an association may increase the risk of these children to develop endocrine disruption, altered pubertal onset, and hormonally-associated cancers.

\section{Methods}

Potential subjects were recruited from the principal investigator's existing patient population at his pediatric primary care office, the Whole Child Center in Oradell, NJ from December 2012 through April 2014. Patients who come to the principal investigator's office for a scheduled checkup were evaluated for eligibility. Subject selection was based on consecutive patients meeting the study criteria. There were 5 boys and 5 girls in each age group, 4-8 years inclusive in the study, meeting inclusion criteria as Tanner Stage 1 (displaying no secondary sexual characteristics on physical exam verified by PI), toilet trained and able to provide a urine sample. Subjects could have no diagnosed hormonally related disorder or cancer and could not be taking any hormonally active medication. At least one parent/guardian needed to be able and willing to give written informed consent.

\section{Recruitment procedures \& informed consent}

Subjects were recruited by the PI (LR), who discussed the study with parents of eligible children. As the study's PI, Dr. Rosen is familiar with the protocol and has received documented education in the protection of human research subjects.

To comply with best practices in ethical approval and consent, this research study was conducted in accordance with the Declaration of Helsinki and was approved prior to the commencement by the HackensackUMC Institutional Review Board (HIRB protocol number Pro00003454). The protocol and de-identified clinical dataset will be made available upon request to any scientist wishing to use them for non-commercial purposes.

After having all questions answered to their satisfaction, parent(s)/guardian(s) who agreed to their child's participation in the study were asked to sign a current, IRB approved informed consent document and HIPAA authorization form. The signed consent document included a statement informing parents/guardians that their child's identity will remain confidential if the results of the trial are published. Pediatric assent was not obtained from subjects prior to participation. 


\section{Lab analysis procedure}

Study kits and lab analysis were provided by Genova Diagnostics (Duluth, GA). Samples were collected at the primary care office with the assistance of a parent/ guardian. The urine sample was collected directly into a glass jar. Approximately $10 \mathrm{ml}$ of urine was liquated from the black-capped glass collection container into the yellow-capped polypropylene plastic vial using the enclosed polyethylene pipette. Room-temperature urine was aliquot immediately into the vial upon the subject's parent or guardian giving the glass collection jar to the research nurse. Both the black-capped collection container and the yellow-capped plastic vial were placed in a biohazard bag with a completed requisition form. Samples were refrigerated immediately after processing until shipment. Samples were shipped overnight in the specimen collection kit box with the frozen ice packs provided to Genova Diagnostics for analysis. The lab used urinary creatinine as a control for concentration and considered samples valid for analysis using creatinine. Limits of detection for the chemicals tested are included in Table 1. Our team confirmed that first morning urine was not necessary for the variables being measured in this study. The following analyses were performed on each sample:

1. Bisphenol A Profile (analysis method: Gas Chromatography/Mass Spectrometry) - Urine: includes Bisphenol A (BPA), 4-Nonylphenol (4NP), and Triclosan

2. Phthalates and Parabens (analysis method: Liquid Chromatography Tandem Mass Spectrometry) Urine: includes metabolites of Di-2-ethylhexyl phthalate (DEHP) which include Mono-(2-ethyl5-hydroxyhexyl) phthalate (MEHHP), Mono-2ethylhexyl phthalate (MEHP), Mono-(2-ethyl-5oxohexyl) phthalate (MEOHP), and Mono-ethyl phthalate (MEtP) and Parabens (Butylparaben, Ethylparaben, Methylparaben, and Propylparaben)

Estronex Estrogen Metabolites (analysis method: Ultra Performance Liquid Chromatography with Tandem Mass Spectrometry) - Urine: 2-Hydroxyestrone (2-OHE1), 2-Hydroxyestradiol (2-OHE2), 2-Hydroxyestrogens $(2-\mathrm{OHE} 1+2)=$ Sum of 2 -Hydroxyestrone $(2-\mathrm{OHE} 1)$ and 2-Hydroxyestradiol (2-OHE2), 2-Methoxyestrone (2-OMeE1), 4-Hydroxyestrone (4-OHE1), 4-Methoxyestrone (4-OMeE1), and 16 alpha-hydroxyestrone (16a-OHE1).

\section{Statistical considerations}

A total of 50 children ( 25 male and 25 female) between 4 and 8 years inclusive were enrolled into the study, with 5 males and 5 females for each age. Additional subjects, selected using the same recruitment procedures, were needed to account for damaged specimen collection or incomplete results due to insufficient urine collected and/or inability of the lab to result all analytes in specific cases. This resulted in a total of 68 subjects enrolled, yielding 50 evaluable subjects. Descriptive statistics and tests were conducted for each year, totaling 5 groups. Results are reported by males only, females only and both genders combined.

For exploratory purposes, summary statistics for the concentrations of each chemical metabolite were computed (minimum, maximum, median and inter-quartile range) for males, for females and for all subjects. These statistics were computed both including and excluding subjects with chemicals below the limits of detection (LOD). When computing summaries and tests with the full data, half the values of the limit of detection were used for these subjects. Table 1 shows these two sets of summary statistics. The proportion of subjects with concentrations below the LOD was calculated for each chemical metabolite. Similar summary statistics were computed for the estrogen metabolites as well.

Comparisons between groups (e.g. males v. females) were assessed using the nonparametric Wilcoxon test, since the data was skewed. The correlation between concentrations of chemical metabolites and estrogen metabolites in prepubescent children were examined by the Spearman's correlation coefficient $(\rho)$. We considered $\rho$ values of $0.60-0.79$ to indicate high correlation, values of 0.40-0.59 moderate, and values of $0.20-0.39$ weak or low correlations. In cases where there are outliers, these were removed and the correlation coefficient recomputed to assess the effect of these outliers. All data analysis in this study was performed using $\mathrm{R}$ and SAS 9.2 (SAS Institute Inc., Cary, NC). Note that we do not formally account for multiple comparisons and report only the unadjusted $\mathrm{p}$ values. The Bonferroni correction is a simple way to correct for multiple comparisons (albeit conservatively). This can be done by the reader by reducing his/her threshold for a significant $p$ value. For example, in this paper, we use a $p$ value of 0.1 . With multiple testing this would require rejection at approximately $0.1 / 10=0.01$ for the roughly 10 environmental chemicals and 10 estrogen variables considered in our study.

\section{Results and discussion}

$100 \%$ of subjects had detectible levels of at least five chemicals in their urine, and $74 \%$ had detectible levels of eight or more chemicals. Of note, $100 \%$ of subjects demonstrated presence of at least one chemical in each class of BPA, phthalates, and parabens. No significant differences were found in mean values between males and females or between age groups for any of the chemicals (Table 1).

$14 \%$ of subjects had all ten chemicals present. Notably, $28 \%$ of subjects had measurable levels of $4 \mathrm{NP}$. 
Table 1 Urinary Chemicals - including and excluding measurements below the limit of detection

\begin{tabular}{|c|c|c|c|c|c|c|c|c|c|c|}
\hline \multicolumn{3}{|l|}{$\begin{array}{l}\mathrm{A}=\text { all } \\
\mathrm{M}=\text { males only } \\
\mathrm{F}=\text { females only }\end{array}$} & \multicolumn{4}{|c|}{$\begin{array}{l}\text { Includes subjects below limits of detection } \\
\text { (replaced with LOD/2) }\end{array}$} & \multicolumn{3}{|c|}{$\begin{array}{l}\text { Excludes subjects below limits } \\
\text { of detection }\end{array}$} & \multirow[t]{2}{*}{$\begin{array}{l}P \text { values for } \\
\text { Wilcoxon Test fo } \\
\text { males } v \text {. females }\end{array}$} \\
\hline 50 Subjects & \# below limit of detection & $\%$ below limit of detection & Min & Median & IQR & Max & Min & Median & IQR & \\
\hline BisphenolA & 35 & 70 & $A=0.05$ & $A=1.12$ & $A=2.83$ & $A=10.80$ & $A=0.17$ & $A=2.05$ & $A=2.2300$ & 0.096 \\
\hline \multirow[t]{2}{*}{$\mathrm{LOD}=0.101 \mathrm{ng} / \mathrm{mL}$} & & & $M=0.05$ & $M=0.58$ & $M=1.78$ & $M=10.80$ & $M=0.40$ & $M=1.61$ & $M=2.715$ & \\
\hline & & & $F=0.05$ & $F=1.64$ & $F=2.12$ & $F=8.63$ & $F=0.17$ & $F=2.35$ & $F=1.955$ & \\
\hline \multirow[t]{3}{*}{ Triclosan LOD = $0.399 \mathrm{ng} / \mathrm{mL}$} & 47 & 94 & $A=0.20$ & $A=18.50$ & $A=112.75$ & $A=2207.00$ & $A=1.00$ & $A=21.00$ & $A=129.5000$ & 0.32 \\
\hline & & & $M=0.20$ & $M=22.00$ & $M=73.00$ & $M=2207.00$ & $M=1.00$ & $M=24.00$ & $M=83.000$ & \\
\hline & & & $F=0.20$ & $F=14.00$ & $F=142.00$ & $F=707.00$ & $F=1.00$ & $F=14.00$ & $F=187.500$ & \\
\hline 4-Nonylphenol & 14 & 28 & $A=0.11$ & $A=0.11$ & $A=0.13$ & $A=6.25$ & $A=0.21$ & $A=0.56$ & $A=1.5375$ & 0.92 \\
\hline \multirow[t]{2}{*}{$\mathrm{LOD}=0.225 \mathrm{ng} / \mathrm{mL}$} & & & $M=0.11$ & $M=0.11$ & $M=0.10$ & $M=6.25$ & $M=0.21$ & $M=0.68$ & $M=1.885$ & \\
\hline & & & $F=0.11$ & $F=0.11$ & $F=0.14$ & $F=4.12$ & $F=0.25$ & $F=0.37$ & $F=0.865$ & \\
\hline MEHHP & 50 & 100 & $A=7.00$ & $A=25.00$ & $A=23.50$ & $A=187.00$ & $A=7.00$ & $A=25.00$ & $A=23.5000$ & 0.95 \\
\hline \multirow[t]{2}{*}{$\mathrm{LOD}=1.022 \mathrm{~g} / \mathrm{L}$} & & & $M=8.00$ & $M=25.00$ & $M=15.00$ & $M=187.00$ & $M=8.00$ & $M=25.00$ & $M=15.000$ & \\
\hline & & & $F=7.00$ & $F=22.00$ & $F=25.00$ & $F=104.00$ & $F=7.00$ & $F=22.00$ & $F=25.000$ & \\
\hline MEHP & 50 & 100 & $A=0.40$ & $A=3.90$ & $A=3.83$ & $A=24.70$ & $A=0.40$ & $A=3.90$ & $A=3.8250$ & 0.56 \\
\hline \multirow[t]{2}{*}{$\mathrm{LOD}=0.199 \mathrm{~g} / \mathrm{L}$} & & & $M=1.00$ & $M=3.80$ & $M=3.60$ & $M=24.70$ & $M=1.00$ & $M=3.80$ & $M=3.600$ & \\
\hline & & & $F=0.40$ & $F=4.00$ & $F=4.00$ & $F=15.20$ & $F=0.40$ & $F=4.00$ & $F=4.000$ & \\
\hline MEOHP & 49 & 98 & $A=0.84$ & $A=13.00$ & $A=10.00$ & $A=76.00$ & $A=4.00$ & $A=13.00$ & $A=10.0000$ & 0.63 \\
\hline \multirow[t]{2}{*}{$\mathrm{LOD}=1.674 \mathrm{~g} / \mathrm{L}$} & & & $M=0.84$ & $M=13.00$ & $M=9.00$ & $M=76.00$ & $M=5.00$ & $M=13.50$ & $M=9.000$ & \\
\hline & & & $F=4.00$ & $F=12.00$ & $F=13.00$ & $F=67.00$ & $F=4.00$ & $F=12.00$ & $F=13.000$ & \\
\hline MetP & 50 & 100 & $A=5.00$ & $A=20.50$ & $A=14.25$ & $A=358.00$ & $A=5.00$ & $A=20.50$ & $A=14.2500$ & 0.28 \\
\hline \multirow[t]{2}{*}{$L O D=0.830 \mathrm{~g} / \mathrm{L}$} & & & $M=5.00$ & $M=18.00$ & $M=8.00$ & $M=358.00$ & $M=5.00$ & $M=18.00$ & $M=8.000$ & \\
\hline & & & $F=5.00$ & $F=22.00$ & $F=14.00$ & $F=162.00$ & $F=5.00$ & $F=22.00$ & $F=14.000$ & \\
\hline Butylparaben & 6 & 12 & $A=0.17$ & $A=0.17$ & $A=0.00$ & $A=5.90$ & $A=0.60$ & $A=1.15$ & $A=1.9250$ & 0.077 \\
\hline \multirow[t]{2}{*}{$\mathrm{LOD}=0.332 \mathrm{~g} / \mathrm{L}$} & & & $M=0.17$ & $M=0.17$ & $M=0.00$ & $M=0.60$ & $M=0.60$ & $M=0.60$ & $M=0.000$ & \\
\hline & & & $F=0.17$ & $F=0.17$ & $F=0.00$ & $F=5.90$ & $F=0.60$ & $F=1.40$ & $F=2.100$ & \\
\hline Ethylparaben & 36 & 72 & $A=0.15$ & $A=0.60$ & $A=1.05$ & $A=8.80$ & $A=0.30$ & $A=0.90$ & $A=0.8000$ & 0.63 \\
\hline \multirow[t]{2}{*}{$L O D=0.298 \mathrm{~g} / \mathrm{L}$} & & & $M=0.15$ & $M=0.50$ & $M=0.95$ & $M=1.90$ & $M=0.30$ & $M=0.90$ & $M=0.775$ & \\
\hline & & & $F=0.15$ & $F=0.60$ & $F=1.05$ & $F=8.80$ & $F=0.40$ & $F=0.85$ & $F=0.775$ & \\
\hline
\end{tabular}


Table 1 Urinary Chemicals - including and excluding measurements below the limit of detection (Continued)

\begin{tabular}{|c|c|c|c|c|c|c|c|c|c|c|}
\hline Methylparaben & 50 & 100 & $A=1.00$ & $A=16.50$ & $A=24.50$ & $A=1000.00$ & $A=1.00$ & $A=16.50$ & $A=24.5000$ & 0.50 \\
\hline \multirow[t]{2}{*}{$\mathrm{LOD}=0.494 \mathrm{~g} / \mathrm{L}$} & & & $M=1.00$ & $M=9.00$ & $M=25.00$ & $M=1000.00$ & $M=1.00$ & $M=9.00$ & $M=25.000$ & \\
\hline & & & $F=2.00$ & $F=19.00$ & $F=22.00$ & $F=267.00$ & $F=2.00$ & $F=19.00$ & $F=22.000$ & \\
\hline Propylparaben & 15 & 30 & $A=1.90$ & $A=1.90$ & $A=3.10$ & $A=124.00$ & $A=5.00$ & $A=8.00$ & $A=6.5000$ & 0.95 \\
\hline \multirow[t]{2}{*}{$\mathrm{LOD}=3.802 \mathrm{~g} / \mathrm{L}$} & & & $M=1.90$ & $M=1.90$ & $M=3.10$ & $M=124.00$ & $M=5.00$ & $M=9.00$ & $M=31.000$ & \\
\hline & & & $F=1.90$ & $F=1.90$ & $F=3.10$ & $F=42.00$ & $F=5.00$ & $F=7.50$ & $F=6.000$ & \\
\hline
\end{tabular}

Note: $p$ values are for tests with those $<$ LOD replaced by LOD/2 
Existing research on 4NP is very limited, with a range of findings. While prior research has documented detectible 4NP levels in adults [12,16, 20], this is the first known published study of 4NP exposure in American children. Interestingly, prior research in a general Belgian population of 131 subjects aged 1 to 75 (average age of 29.6) did not find 4NP in detectible levels in the urine samples analyzed [15]. Another study in $11 \mathrm{hu}-$ man tissue samples of patients aged between 9 and 62 years (average age of 34 years), found mostly undetectable levels of 4NP and concluded the chemical is not of significant importance for human exposure [17]. However, a study measuring nonylphenol in Taiwanese children aged 6.5-15.0 years found NP in 84 to $94 \%$ of samples measured for NP concentrations of 10-250 ng/ $\mathrm{mL}$ [19]. The wide variation of levels documented in existing research coupled with our finding that $4 \mathrm{NP}$ is present in nearly $1 / 3$ of this pediatric population suggests a need for further evaluation of this ubiquitous chemical.

Analysis of the estrogen variables shows there were no significant differences by age. As there were no zero values for estrogen metabolites, all were above the limit of detection. Upon analysis by gender, females have larger maximum, standard deviations and slightly larger means for 2-OHE2 and 2-OHE1 + 2 (Table 2). 16a-OHE1 was the only estrogenic metabolite significantly different (higher, in this case) in girls than boys, with a $p$ value of 0.013 from the Wilcoxon test. Elevated levels of this metabolite are linked to increased risk and worse prognosis for estrogen-sensitive cancers and autoimmune conditions [30], though the specific levels measured in this study are not directly associated with known health risks. It is not known if girls simply have relatively higher levels of $16 \mathrm{a}-\mathrm{OHE} 1$ than boys.

Since the data is skewed and non-normal, we use the Spearman's correlation coefficient $(\rho)$ to assess the correlations between estrogen markers and chemicals. Using a test to assess whether $\rho$ is zero or not [31], we found that none of the correlations were statistically significant at the 0.1 level after accounting for multiple testing.

There was a widely divergent single outlier identified for most chemicals: the $22^{\text {nd }}$ male had high values of triclosan, $21^{\text {st }}$ male of $4 \mathrm{NP}$ and the $23^{\text {rd }}$ male of MetP. The $23^{\text {rd }}$ female had high values of $4 \mathrm{NP}$ and propylparaben, and the $24^{\text {th }}$ female of ethylparaben. It would be interesting to explore the possibility of subpopulations that are particularly at risk of higher exposures to these chemicals and/or increased risk for health effects from exposures. But with only 3 such subjects we are not able to do this for our data. Therefore, this pilot study suggests the need for additional research with a larger population.

\section{Limitations}

A limitation of this study was the small sample size (50), with only 5 boys and 5 girls in each age group. There is an instance of an extremely large chemical outlier value. This outlier greatly affected the correlation coefficient between chemical concentrations and estrogen metabolism markers. All the other data points showed no correlation between the variables. Further investigation into the measurement process would need to be done to

Table 2 Summary of estrogen metabolites

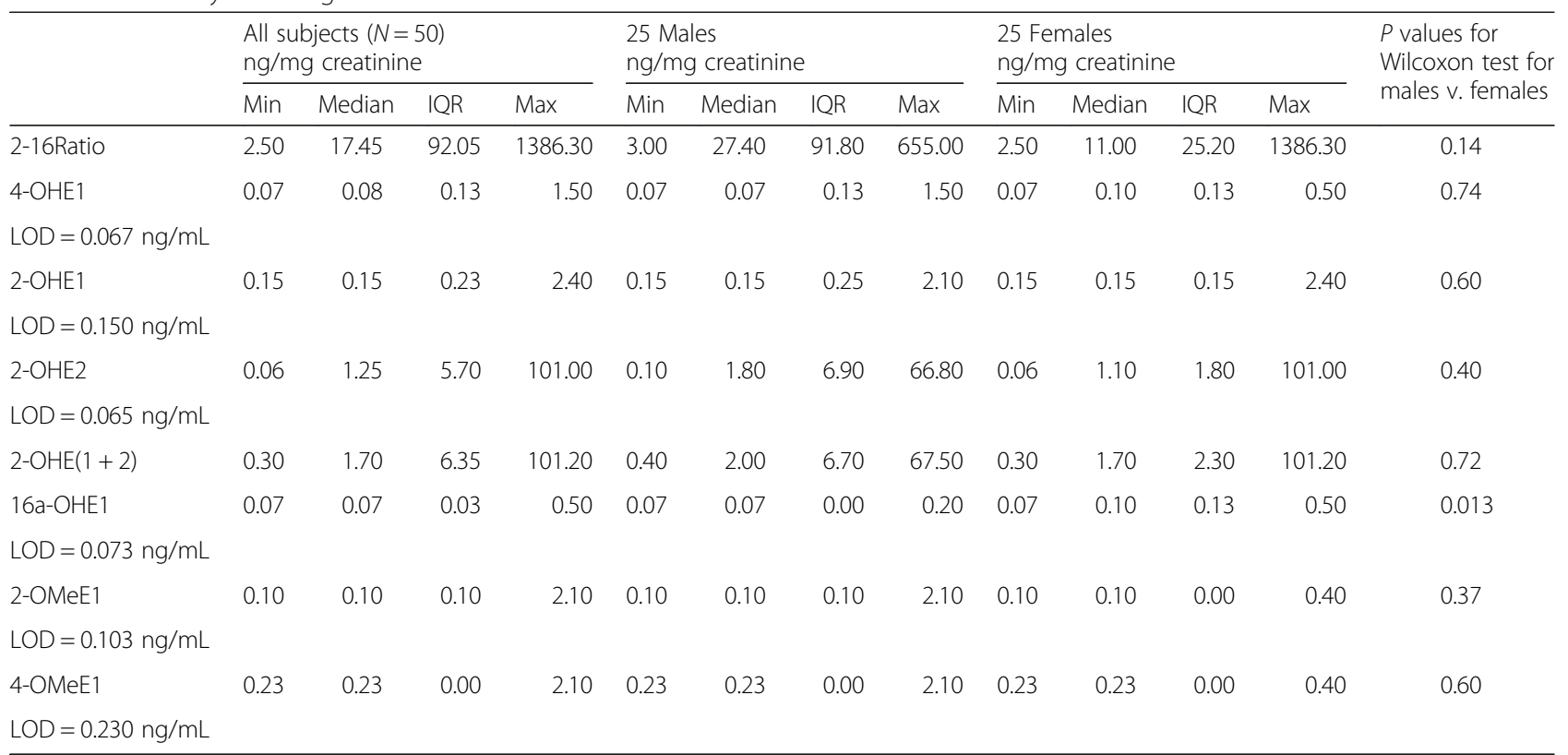


determine if the outlier value is spurious. It must be noted that even if the value is not spurious, an inference cannot be drawn based on one observation. The small sample size may be a reason we are unable to determine any correlation between chemical concentrations and estrogen metabolism markers. Considering the lack of statistically significant difference in estrogen metabolism between boys and girls in this study, it may be that prepubertal children do not demonstrate this correlation while post-pubertal adolescents and adults do, perhaps as a function of the low estrogen values in pre-pubertal children. Additionally, after pubertal onset, the associations may be sexually dimorphic, as has been observed in some pediatric cohorts [19].

Further research evaluating larger samples sizes of pre- and post-pubertal subjects would be of value, given the presence of eight or more hormonally disruptive chemicals in nearly three-fourths of subjects.

\section{Conclusions}

Endocrine disrupting environmental chemicals were detected in all children in the study, and nearly threequarters of subjects had detectible levels of eight or more chemicals. Nearly $1 / 3$ of subjects had measurable levels of $4 \mathrm{NP}$ in their urine, a novel finding, suggesting a need for more research regarding the presence and impact of this chemical. After the removal of outliers, no associations were found between the urine levels of chemicals tested and estrogen metabolites. However, the presence of multiple chemicals in a majority of children's urine coupled with increasing prevalence of pediatric hormonal disorders warrants further research to elucidate potential causal mechanisms in pre- and post-pubertal children.

\section{Abbreviations}

APs: alkylphenols; APEs: alkylphenolethoxylates; BPA: Bisphenol A; DIEHC: The Deirdre Imus Environmental Health Center ${ }^{\oplus ;}$ 4NP: 4-Nonylphenol; DEHP: Di-2ethylhexyl phthalate; MEHHP: Mono-(2-ethyl-5-hydroxyhexyl) phthalate; MEHP: Mono-2-ethylhexyl phthalate; MEOHP: Mono-(2-ethyl-5-oxohexyl) phthalate; MEtP: Monoethyl phthalate;

2-OHE1: 2-Hydroxyestrone; 2-OHE2: 2-Hydroxyestradiol; 16a-OHE1:

16a-Hydroxyestrone; 4-OHE1: 4 Hydroxyestrone; 2-OMeE1: 2-Methoxyestrone; 4-OMeE1: 4-Methoxyestrone.

\section{Competing interests}

The authors declare that they have no competing interests.

\section{Authors' contributions}

ESI participated in the design of the study, study oversight \& drafted the manuscript. JML performed the statistical analysis and drafted the statistical portions of the manuscript. LR participated in the design of the study, contributed medical expertise and manuscript preparation and detailed manuscript review. All authors read and approved the final manuscript.

\section{Acknowledgements}

This study was funded and conducted by The Deirdre Imus Environmental Health Center $^{\circledast}$ (DIEHC) at Hackensack University Medical Center. The research team would like to thank Research Nurse Karen Overgaard and interns Nikita Manavi and Lucerito Ruiz for their expertise and dedication.

\section{Standards of reporting}

The STROBE Checklist was consulted during the review of this manuscript.

\section{Author details}

${ }^{1}$ The Deirdre Imus Environmental Health Center ${ }^{\circledast}$, Hackensack University Medical Center, 30 Prospect Ave, Research Building, Hackensack, NJ 07601 USA. ${ }^{2}$ Department of Mathematical Sciences, NJ Institute of Technology, University Heights, Newark, NJ 07102, USA

\section{Received: 25 February 2015 Accepted: 11 December 2015} thon

\section{References}

1. National Cancer Institute Fact Sheet: Cancer in Children and Adolescents. [http://www.cancer.gov/cancertopics/factsheet/Sites-Types/childhood]. Accessed 8 Aug 2014

2. Ward E, DeSantis C, Robbins A, Kohler B, Jemal A. Childhood and adolescent cancer statistics. CA Cancer J Clin. 2014;64(2):83-103.

3. Biro FM, Galvez MP, Greenspan LC, Succop PA, Vangeepuram N, Pinney SM, et al. Pubertal assessment method and baseline characteristics in a mixed longitudinal study of girls. Pediatrics. 2010;126(3):e583-90.

4. President's Cancer Panel 2008-2009 Annual Report: Reducing Environmental Cancer Risk: What We Can Do Now. [http://deainfo.nci.nih. gov/advisory/pcp/annualReports/pcp08-09rpt/PCP_Report_08-09_508.pdf]. Accessed 8 Aug 2014

5. Wolff MS, Teitelbaum SL, Pinney SM, Windham G, Liao L, Biro F, et al. Investigation of relationships between urinary biomarkers of phytoestrogens, phthalates, and phenols and pubertal stages in girls. Breast Cancer and Environment Research Centers. Environ Health Perspect. 2010; 118(7):1039-46.

6. Gee RH, Charles A, Taylor N, Darbre PD. Oestrogenic and androgenic activity of triclosan in breast cancer cells. J ApplToxicol. 2008;28(1):78-91.

7. Li X, Ying GG, Zhao JL, Chen ZF, Lai HJ. Su HC.4-Nonylphenol, bisphenol-A and triclosan levels in human urine of children and students in China, and the effects of drinking these bottled materials on the levels. Environ Int 2013;52:81-6.

8. Calafat AM, Ye X, Wong LY, Reidy JA, Needham LL. Exposure of the U.S population to bisphenol A and 4-tertiary-octylphenol: 2003-2004. Environ Health Perspect. 2008;116(1):39-44.

9. Lankester J, Patel C, Cullen MR, Ley C, Parsonnet J. Urinary triclosan is associated with elevated body mass index in NHANES. PLoS One. 2013; 8(11):e80057.

10. Crinnion WJ. Toxic effects of the easily avoidable phthalates and parabens. Altern Med Rev. 2010:15(3):190-6.

11. Breast Cancer Fund: Chemicals and Radiation Linked to Breast Cancer Alkylphenols. [http://www.breastcancerfund.org/clear-science/radiationchemicals-and-breast-cancer/alkylphenols.html]. Accessed 20 Nov 2015.

12. Calafat AM, Kuklenyik Z, Reidy JA, Caudill SP, Ekong J, Needham LL. Urinary concentrations of bisphenol $A$ and 4-nonylphenol in a human reference population. Environ Health Perspect. 2005;113(4):391-5.

13. Colón I, Caro D, Bourdony CJ, Rosario O. Identification of phthalate esters in the serum of young Puerto Rican girls with premature breast development. Environ Health Perspect. 2000;108(9):895-900

14. Chen M, Tang R, Fu G, Xu B, Zhu P, Qiao S, et al. Association of exposure to phenols and idiopathic male infertility. J Hazard Mater. 2013;250-251:115-21

15. Pirard C, Sagot C, Deville M, Dubois N, Charlier C. Urinary levels of bispheno A, triclosan and 4-nonylphenol in a general Belgian population. Environ Int. 2012;48:78-83.

16. Gyllenhammar I, Glynn A, Darnerud PO, Lignell S, van Delft R, Aune M 4-Nonylphenol and bisphenolA in Swedish food and exposure in Swedish nursing women. Environ Int. 2012;43:21-8.

17. Geens T, Neels H, Covaci A. Distribution of bisphenol-A, triclosan and n-nonylphenol in human adipose tissue, liver and brain. Chemosphere. 2012:87(7):796-802.

18. Chen GW, Ding WH, Ku HY, Chao HR, Chen HY, Huang MC, et al. Alkylphenols in human milk and their relations to dietary habits in central Taiwan. Food ChemToxicol. 2010:48(7):1939-44.

19. Hou JW, Lin CL, Tsai YA, Chang CH, Liao KW, Yu CJ, et al. The effects of phthalate and nonylphenol exposure on body size and secondary sexual characteristics during puberty. Int J Hyg Environ Health. 2015;218(7):603-15. 
20. Lopez-Espinosa MJ, Freire C, Arrebola JP, Navea N, Taoufiki J, Fernandez MF, et al. Nonylphenol and octylphenol in adipose tissue of women in Southern Spain. Chemosphere. 2009;76(6):847-52.

21. Calafat AM, Ye X, Wong LY, Bishop AM, Needham LL. Urinary concentrations of four parabens in the U.S. population: NHANES 2005-2006. Environ Health Perspect. 2010;118(5):679-85.

22. Wolff MS, Teitelbaum SL, McGovern K, Windham GC, Pinney SM, Galvez M, et al. Breast Cancer and Environment Research Program: Phthalate exposure and pubertal development in a longitudinal study of US girls. Hum Reprod. 2014;29(7):1558-66.

23. Park MA, Choi KC. Effects of 4-nonylphenol and bisphenol A on stimulation of cell growth via disruption of the transforming growth factor- $\beta$ signaling pathway in ovarian cancer models. Chem Res Toxicol. 2014;27(1):119-28.

24. Khanna S, Dash PR, Darbre PD. Exposure to parabens at the concentration of maximal proliferative response increases migratory and invasive activity of human breast cancer cells in vitro. J ApplToxicol. 2014;34(9):1051-9.

25. Boberg J, Taxvig C, Christiansen S, Hass U. Possible endocrine disrupting effects of parabens and their metabolites. ReprodToxicol. 2010;30(2):301-12.

26. Henry ND, Fair PA. Comparison of in vitro cytotoxicity, estrogenicity and anti-estrogenicity of triclosan, perfluorooctane sulfonate and perfluorooctanoic acid. J ApplToxicol. 2013;33(4):265-72.

27. Bonefeld-Jørgensen EC, Long M, Hofmeister MV, Vinggaard AM. Endocrinedisrupting potential of bisphenolA, bisphenol A dimethacrylate, 4-nnonylphenol, and 4-n-octylphenol in vitro: new data and a brief review. Environ Health Perspect. 2007;115 Suppl 1:69-76.

28. Kabat GC, O'Leary ES, Gammon MD, Sepkovic DW, Teitelbaum SL, Britton JA, et al. Estrogen metabolism and breast cancer. Epidemiology. 2006;17(1):80-8.

29. Sepkovic DW, Bradlow HL. Estrogen hydroxylation-the good and the bad. Ann NY Acad Sci. 2009;1155:57-67.

30. Genova Diagnostics Essence Hormone Tests: Estrogen Metabolism Assessment. [https://www.gdx.net/core/one-page-test-descriptions/ Estrogen-Metabolism-Assess-Test-Description.pdf]. Accessed 14 Nov 2015.

31. Hollander M, Wolfe DA. Nonparametric Statistical Methods. New York: John Wiley \& Sons; 1973. p. 185-94.

\section{Submit your next manuscript to BioMed Central and we will help you at every step:}

- We accept pre-submission inquiries

- Our selector tool helps you to find the most relevant journal

- We provide round the clock customer support

- Convenient online submission

- Thorough peer review

- Inclusion in PubMed and all major indexing services

- Maximum visibility for your research

Submit your manuscript at www.biomedcentral.com/submit

) Biomed Central 